\title{
A Review Paper on Semiconductors using Big Data
}

\author{
Yash Shukla \\ M.Tech - Information Technology \\ Parul University \\ Waghodia, Vadodara, India
}

\begin{abstract}
In the Digital world there is arrival of new type of modifications. Semiconductors faced much kind of new difficulties through which the data management is very much complicated. Here "Big data" is applied to the system which results in the flexibility and speed of the manufacturing of the process. Big data will overtake the critical risk and provide friendly platform systems which are useful for the construction of the semi-conductors. To manufacture a good and stabilized semiconductor a well type of Fault Detection and Classification (FDC) has been required using big data.
\end{abstract}

\section{Keywords}

Big Data, Semiconductor, Hadoop.

\section{INTRODUCTION}

The use of Digital verse is been doubling these years. Excessive use has lead to the different type of updates and modification in the system. As requirements are rapidly growing in semiconductors based on data types, volumes, analytics, we are faced with new different new approaches. Various different types of new and modified types of applications are also been required. Here the Fault Detection and classification (FDC) is been put to use along with the use of cooperating type of Manufacturing Equipment System (MES). After the use of these types of applications in the manufacturing of Semiconductor the use of the control such as Run to Run (R2R) function is also done. There are also various types of Impacts which will used to enable more effectiveness such as Predictive Maintenance (PDM), Predictive scheduling, Virtual Metrology (VM) and yield prediction.

\section{APPROACH}

\subsection{Involvement of Various Components through Big Data}

Semiconductor can be manufactured through many approaches and methods using the big data. For these Hadoop System can be considered. Its main goal or impact will be to better enable more and more use of predictive technologies like Predictive Maintenance and Predictive Scheduling into it. It would also include the various types of components like: Apache Hadoop: It provides an open source framework for the designing purposes and also for the storage activities.

Hadoop Distributed Filing System (HDFS): It is used for processing large data and also used for availability purpose. Data Warehousing: It is used for extensible storage capacity[2]

\subsection{Involvement of different types of systems through Big Data}

There are also various kind of different type of systems which are required for the manufacturing of semi conductors such as Run to Run (R2R) and Fault Detection (FD) Techniques.

These Components are used to store the extensive data collection and also used for managing the environment. These configurations generally used the Traditional and Pre Hadoop Technologies for the configuration processes.

Various types of equipment's and parts are been used for the designing for the creation of the semi conductor. This involve in the creation of the semi conductor in an efficient manner. They undergo the process of the execution in the system.

It also includes the different types such as:

Manufacturing Execution System[MES], Equipment[EQ], Monitoring Tool[MT], Fault Detection and Classification[FDC], Virtual Metrology[VM].

\subsection{Requirement used for Manufacturing} Purposes:

There are following requirements used for the manufacturing purposes:

- Data Collection and Management.

- Data Analysis.

- Data Utilization.

- Data Collection and Management:

There has been high use of FDC (Fault Detection Control) in it. Here High speed FDC Judgments and system response is used to carry out each wafer. Here the conventional process is a batch process which is used to carry out the Raw Data which is further stored in the Data Base (DB).

The Occurrence of the new handling should be started when the extraction and fully use of the previous data is been done. If the handling process is not done properly then there is an chance of delay in the database reading process. In order to perform the system fully in an efficient way and to use the storage capacity in a better way, the implementation of data compression as well as the archive function can be done by the storage of the data amount which can be further kept as a constant.

Here FDC mainly deals with fault detection of each wafer and it is also used for the system response. 


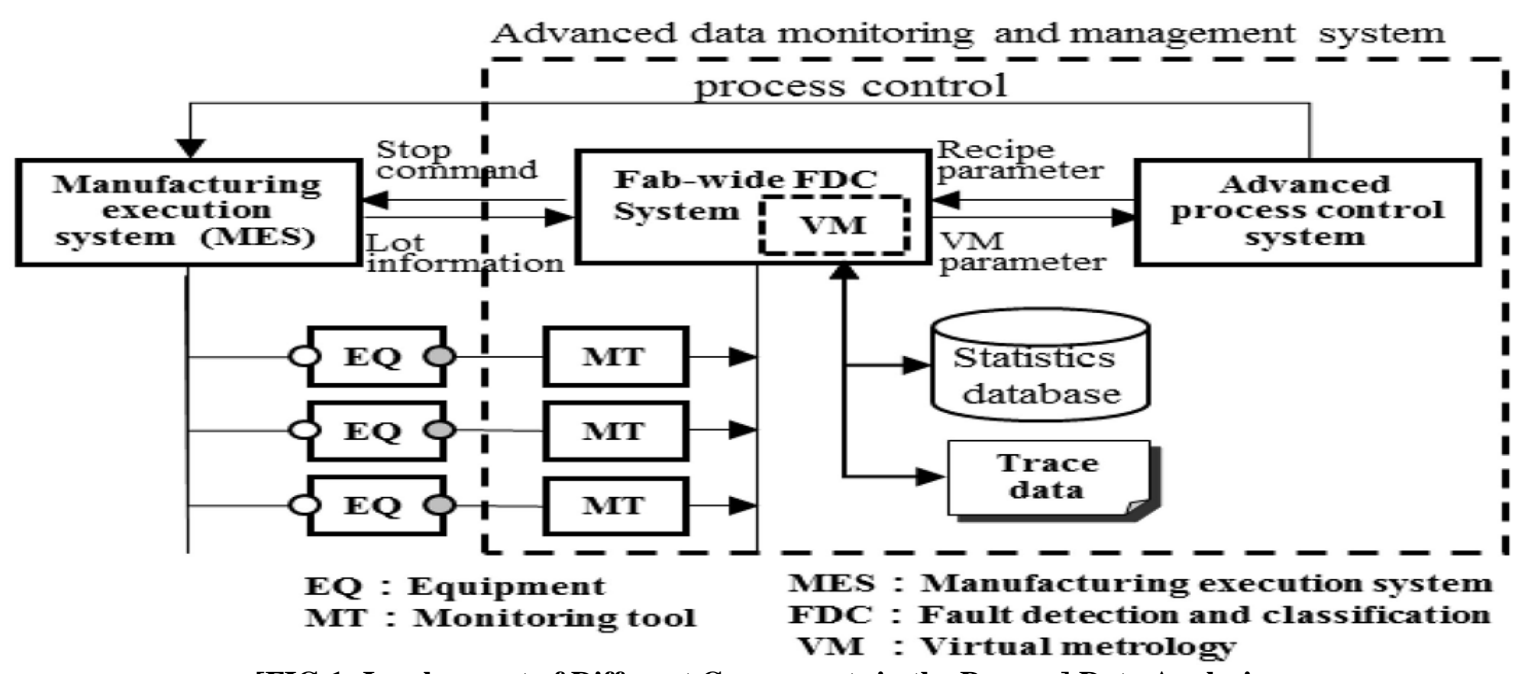

[FIG.1: Involvement of Different Components in the Process] Data Analysis

In Data Analytical Method the different types of variables are been correlated. It takes huge amount of data to prepare the different types of variable data sheet which contains different types of equipment and parameters.

Sometime due to excessive load more effort is been required to collect the parameters in it. Therefore we are required to solve this problem. To provide an optimal

solution to these problems the development of data aggression and merging of multiple functions can be done easily. This can be used as a conventional method for solving the problem.

\section{- Data Utilization}

Here the performance is measured on the basis of the no of processes being carried out in the manufacturing of the semiconductor. Here there is a usage of conventional APC as well as V-APC which is been required[1]

\section{CONCLUSION:}

There could be an opportunity or chances that further the significant use of APC system can be used more and more and also some modifications can be done in it. Here the use of $\mathrm{R} 2 \mathrm{R}$ can also be done and necessary changes can be applied in it.

\section{REFERENCES}

[1] Advanced semiconductor Manufacturing using Big data.(2015) Tomio Tsuda, Shinji Inoue

[2] Big data applied to Semiconductor Manufacturing Advanced Process Control.(2016) James Moyne, Jamini Samantaray. 